\title{
Detection of prostate specific antigen in serum at the femto-gram per milliliter level using the intrinsic amplification of a field-effect enzymatic immuno-sensing system
}

\author{
Jiapeng Wang \\ Cleveland State University \\ Siu-Tung Yau \\ Cleveland State University, s.yau@csuohio.edu
}

Follow this and additional works at: https://engagedscholarship.csuohio.edu/enece_facpub How does access to this work benefit you? Let us know!

\section{Publisher's Statement}

NOTICE: this is the author's version of a work that was accepted for publication in Electrochimica Acta. Changes resulting from the publishing process, such as peer review, editing, corrections, structural formatting, and other quality control mechanisms may not be reflected in this document. Changes may have been made to this work since it was submitted for publication. A definitive version was subsequently published in Electrochimica Acta, 111, , (07-05-1905); 10.1016/j.electacta.2013.08.046

\section{Repository Citation}

Wang, Jiapeng and Yau, Siu-Tung, "Detection of prostate specific antigen in serum at the femto-gram per milliliter level using the intrinsic amplification of a field-effect enzymatic immuno-sensing system" (2013). Electrical Engineering \& Computer Science Faculty Publications. 270.

https://engagedscholarship.csuohio.edu/enece_facpub/270 


\title{
Detection of prostate specific antigen in serum at the femto-gram per milliliter level using the intrinsic amplification of a field-effect enzymatic immuno-sensing system
}

\author{
Jiapeng Wang ${ }^{\mathrm{a}}$, Siu-Tung Yau ${ }^{\mathrm{a}, \mathrm{b}, *}$ \\ Department of Electrical and Computer Engineering, Cleveland State University, Cleveland, $\mathrm{OH} 44115$, USA \\ ${ }^{b}$ The Applied Bioengineering Program, Cleveland State University, Cleveland, $\mathrm{OH} 44115, \mathrm{USA}$
}

\section{Introduction}

The ultimate goal of biomarker-based cancer-screening assays is to detect cancers at a stage early enough so that treatment is likely to be successful. Prostate-specific antigen (PSA) is a serine protease produced by the prostate epithelium [1,2]. Although it has been the subject of debate as a biomarker for routine screening of prostate cancer [3], PSA can be used as an unambiguous indicator of prostate cancer recurrence after prostatectomy [4,5]. Monitoring PSA level after radical prostatectomy allows earlier intervention with adjuvant radiation or endocrine therapy. Studies show that in patients treated with post-prostatectomy adjuvant radiation therapy, the patients with low PSA levels at the beginning of the treatment had the best response to the therapy [6,7]. However, at extremely low levels, PSA may fluctuate and, therefore, ultrasensitive detection capabilities are needed to quantify the trend of the fluctuation.

Two recently developed methods for ultrasensitive detection of PSA should be noted. The first method employed bio-functionalized gold nanoparticles (Au-NP) as the probe for bar-code assay [8]. Au-NP was used to generate the PSA-specific antibody-Au-NP

\footnotetext{
* Corresponding author at: Department of Electrical and Computer Engineering, Cleveland State University, Cleveland, OH 44115, USA. Tel.: +1 216875 9783; fax: +1 2166875405 .

E-mail address: s.yau@csuohio.edu (S.-T. Yau).
}

probes. Magnetic microparticles (MMP), functionalized with PSAspecific antibodies, were mixed with the serum PSA sample and subsequently with the antibody-Au-NP probes to form the antibody-PSA-antibody sandwich structure. After magnetic separation, the PSA barcodes were detected using the scanometric assay, which took advantage of Au-NP catalyzed silver enhancement. The detection limit of this method was about $330 \mathrm{fg} \mathrm{mL}-1$ serum, obtained with a sample size of $<100 \mu \mathrm{L}$. The second method employed multi-label secondary antibody-nanotube bioconjugates to detect PSA in serum and tissue lysates using a conventional electrochemical immunosensor [9]. Amplified detection signal was obtained as the result of using bioconjugates made by linking horseradish peroxidase (HRP) labels and secondary antibodies (Ab2) to carbon nanotubes at high HRP/Ab2 ratio. The electron transfer mediator hydroquinone was used to facilitate electron transfer. This method provided a detection limit of $4 \mathrm{pg} \mathrm{mL}^{-1}$, obtained with PSA in $10 \mu \mathrm{L}$ of undiluted calf serum, featuring a mass detection limit of $40 \mathrm{fg}$. The ultra low detection limits provided by the two methods was the result of increasing the amount of the labeling substance, leading to extrinsic amplification.

Recently, an ultrasensitive enzymatic electrochemical detection method has been demonstrated with pico-molar (pM) detection limits for molecular analytes [10]. This field-effect enzymatic detection (FEED) method employs an external gating voltage to provide intrinsic amplification of the signal current by inducing an interfacial electric field to modulate interfacial charge transfer 


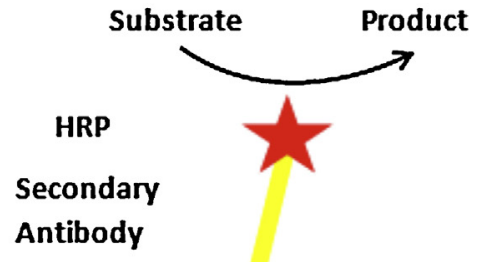

Antibody

PSA

Primary

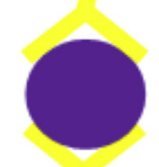

Antibody

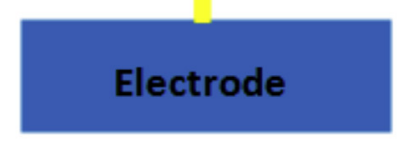

(a)

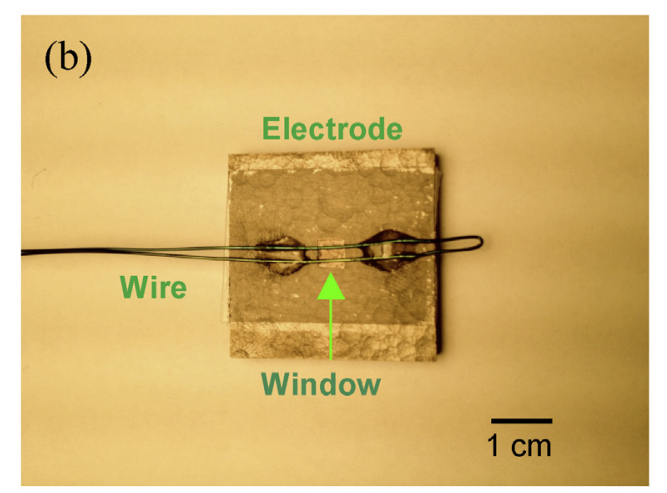

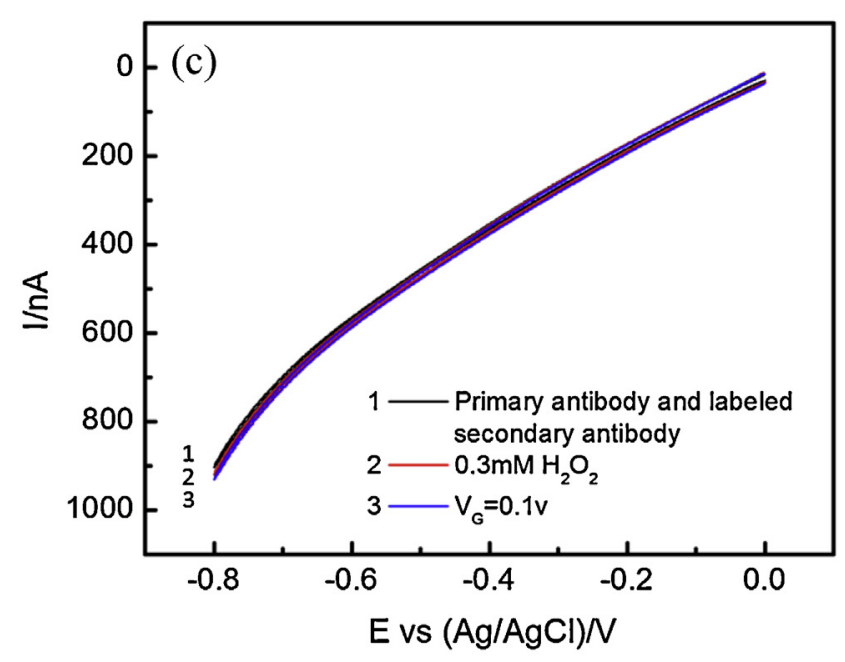

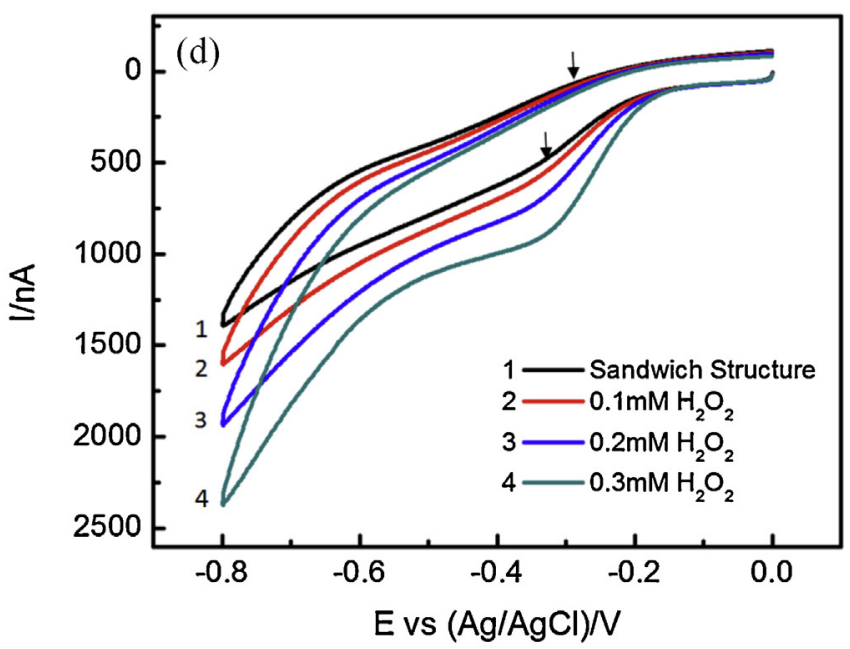

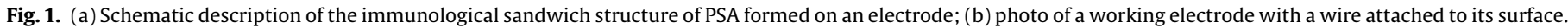

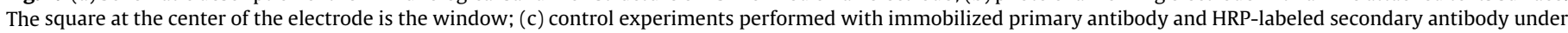

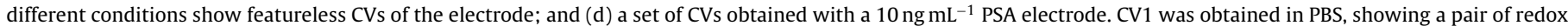
peaks indicated by the arrows. CV2-CV4 were obtained with the same electrode in different $\mathrm{H}_{2} \mathrm{O}_{2}$ concentrations.

[10]. In this article, we describe the ultrasensitive detection of PSA achieved by applying FEED to the conventional amperometric immunosensing scheme, in which the nanometer-sized antibody-antigen-antibody (Ab-Ag-Ab) sandwich structure is formed on an electrode as shown in Fig. 1(a). The intrinsic amplification provided by FEED has allowed us to detect PSA in serum samples with $27 \mathrm{fg} \mathrm{mL}^{-1}$ detection limit with $20 \mathrm{fg} \mathrm{mL}^{-1}$ detection resolution. Note that electron transfer through the $\mathrm{Ab}-\mathrm{Ag}-\mathrm{Ab}$ nanostructure in immuno-sensing systems is an overlooked problem, although amperometric signals (electric currents) traveling through the $\mathrm{Ab}-\mathrm{Ag}-\mathrm{Ab}$ structure has been observed in the detection of biomarkers. Our results indicate the feasibility of using FEED to control electron transfer through the nanostructure, leading to an ultralow detection limit for PSA. The electrochemical nature of the detection and the ultralow detection of PSA in serum suggest the possibility of integration of the detection system with microfluidics on chips, leading to the point-of-care approach for early detection of recurrence of prostate cancer after prostatectomy [11].

\section{Experimental}

\subsection{FEED system}

The principle of FEED has been explained and the detection system described previously [10]. Briefly, the detection system consists of a conventional three-electrode electrochemical cell modified with additional gating electrodes for applying a gating voltage $V_{\mathrm{G}}$ to the detecting (working) electrode, on which a redox enzyme, the sensing element, is immobilized. $V_{G}$ rearranges ions in the sample solution at the electrode-solution interface, inducing an electric field that penetrates the immobilized enzyme. The field reduces the effective height of the tunnel barrier between the active site of the enzyme and the electrode, therefore increasing the tunnel rate of electrons and resulting in intrinsic amplification of the signal current. A detailed description of the FEED-immunosensing system used in this work is provided in the Appendix (Supplementary Material). In the present application of FEED, the enzyme is immobilized on the electrode via the sandwich immune complex. 


\subsection{Reagents, electrodes and measurements}

PSA samples and antibodies used in this work were purchased from ANOGEN (Mississauga, Canada). Monoclonal (mouse) antihuman PSA antibody (clone number CHYH1) was used as the primary antibody, and polyclonal anti-human PSA antibody (clone number $\mathrm{CHYH} 2$ ) labeled with horseradish peroxidase (HRP) was used as the secondary antibody. PSA standard solutions in calf serum and the control sample (serum without PSA) were used in detection and control measurements. Disks of pyrolytic graphite $(\mathrm{PG})(3 \mathrm{~cm} \times 3 \mathrm{~cm})$ were used as electrodes. A layer of the conducting polymer, polyaniline (PANI), was deposited on PG electrodes to host antibodies on electrodes [12] and to be used as electron mediator, shuttling electrons between HRP and the electrode [13]. The PANI layer was synthesized on the electrode using electrochemical polymerization of aniline in a solution of $0.1 \mathrm{M}$ aniline and $1 \mathrm{M} \mathrm{HCl}$ at a potential of $1.2 \mathrm{~V}$ for $100 \mathrm{~s}$ [14]. The PANI prepared using this method was used previously in a HRP-based biosensor to detect $\mathrm{H}_{2} \mathrm{O}_{2}$ at $\mathrm{pH} 6.8$ [15] as well as in a pH sensor [16] because the conductivity of PANI persists up to $\mathrm{pH}$ 9. Alternatively, sulfonated PANI was prepared by adding poly(vinylsulfonic acid) (Aldrich, cat. \# 27,842-4) to aniline and $\mathrm{HCl}$ during electrochemical polymerization as described previously [17], to increase the redox activity of PANI at $\mathrm{pH}$ 7. The results shown in this work were obtained with PANI prepared using $\mathrm{HCl}$ only while the two kinds of PANI led to similar results. Glutaraldehyde was used as a cross-linker, coupling the primary antibody to the highly porous PANI so that the Ab-PSA-Ab(HRP) structure was firmly entrapped in PANI and the HRP was in contact to PANI. Previous XPS analysis [12] shows that glutaraldehyde binds to the amine groups on PANI to form an imine bond.

A plastic mask with a $2 \mathrm{~mm} \times 2 \mathrm{~mm}$ to $3 \mathrm{~mm} \times 3 \mathrm{~mm}$ opening was used to define a window on the PANI layer. The window was further modified by depositing $2 \mu \mathrm{L}$ of glutaraldehyde $(25 \%$ with water, diluted 100 times with water) on PANI until dry. PSAdetecting electrodes were formed by incubating the modified PG electrodes with $10 \mu \mathrm{L}$ of $1 \mathrm{mg} / \mathrm{mL}$ primary antibody dissolved in phosphate buffered saline (PBS) for $1 \mathrm{~h}$ at room temperature and a subsequent rinse with de-ionized water. To detect PSA, a PSAdetecting electrode was incubated with $5-10 \mu \mathrm{L}$ of a PSA sample (a PSA standard serum solution) for $1 \mathrm{~h}$ at $37^{\circ} \mathrm{C}$. After rinsing with de-ionized water and blocking possible open areas on the electrode with bovine serum albumin ( $40 \mathrm{mg} / \mathrm{mL}$ diluted to $10 \%$ in Dulbecco's phosphate-buffered saline), the same electrode was incubated with $5-10 \mu \mathrm{L}$ of $0.5 \mu \mathrm{g} / \mathrm{mL}$ HRP-labeled secondary antibody for $30 \mathrm{~min}$ at $37^{\circ} \mathrm{C}$ and then rinsed with de-ionized water. The Ab-PSA-Ab(HRP) sandwich structure now has formed on the electrode. Finally, the electrode was now formed as the working electrode for electrochemical measurements. A piece of $0.5 \mathrm{~mm}$-diameter copper wire coated with a thin layer of insulator (enamel) was used as the gating electrode for applying $V_{\mathrm{G}}$. The wire was bent to form a U-shaped structure and was attached on the sensing electrode using nonconductive epoxy. Fig. 1(b) shows a working electrode with a wire attached to its surface. All PSA samples were prepared with undiluted serum sample as received. Note that, for each data point on the calibration curves shown in this work, 5 electrodes were made and tested for proper behaviors. Generally, 3 or 4 electrodes showed the proper behaviors such as the presence the redox peaks of the enzyme and were used to determine the data point.

Electrochemical measurements made using a conventional three-electrode electrochemical cell were used to extract the detection signal. A commercial $\mathrm{Ag} / \mathrm{AgCl}(3 \mathrm{M} \mathrm{KCl})$ electrode was used as the reference electrode, and a platinum wire was used as the counter electrode. The volume of the electrochemical cell was $1 \mathrm{~mL}$. The cell was driven by a commercial electrochemical controller (CHI $660 \mathrm{C}$ Work Station). A potential scan rate of $20 \mathrm{mV} / \mathrm{s}$ was used in recording cyclic voltammograms (CV) and linear voltammograms (LV). PBS (0.1 M at $\mathrm{pH} 7)$ was prepared using de-ionized water $(18.2 \mathrm{M} \Omega \mathrm{cm})$. Commercially available hydrogen peroxide (Fisher scientific, 30\% concentration) was diluted to the concentrations used in the experiment. All measurements were made with deaerated PBS at room temperature. Reproducible results were obtained by repeating each measurement multiple times.

\section{Results and discussion}

The detection of PSA was achieved by applying FEED to the conventional amperometric immuno-sensing method, the operation of which requires the sandwich structure, Ab-PSA-Ab(HRP), to be formed on the PSA-detecting electrode as shown in Fig. 1(a). Control experiments were performed using electrodes immobilized with different participants of the immuno-reaction in order to avoid misinterpretation of experimental results. Fig. 1(c) shows a set of cyclic voltammograms (CVs) obtained under different conditions with a PG electrode modified with PANI and glutaraldehyde as described above. The modified electrode was first immobilized with the primary $\mathrm{Ab}$ and subsequently with the labeled secondary $\mathrm{Ab}(\mathrm{HRP})$ followed by a rinse to remove unstable structures from the electrodes. CV1 and CV2 were obtained respectively with this electrode in PBS only and PBS with the presence of $0.3 \mathrm{mM} \mathrm{H}_{2} \mathrm{O}_{2}$. Note that $\mathrm{H}_{2} \mathrm{O}_{2}$ is reduced to water by the catalytic activity of HRP [18]. CV3 was obtained with the same electrode in $\mathrm{H}_{2} \mathrm{O}_{2}$ when a $V_{\mathrm{G}}$ of $0.3 \mathrm{~V}$ was applied. The three CVs appear to be featureless and have similar magnitudes. Similar results were obtained with the modified electrode, which was immobilized first with PSA and subsequently with secondary $\mathrm{Ab}(\mathrm{HRP})$ to form the PSA-Ab(HRP) immuno-complex. Additional control experiments using graphite electrodes without PANI but with glutaraldehyde and the sandwich immuno-complex resulted in no observable signal.

Fig. 1(d) shows a set of CVs obtained with a modified electrode, which was further processed according to the procedures required to form the Ab-PSA-Ab(HRP) structure as described above. The concentration of PSA used to prepare this electrode was $10 \mathrm{ng} \mathrm{mL}^{-1}$. CV1 was obtained in PBS, showing a pair of redox peaks as indicated by the arrows with a formal potential of $-0.3 \mathrm{~V} \mathrm{vs.} \mathrm{Ag} / \mathrm{AgCl}$. Previous works on the direct electron transfer associated with immobilized HRP show that the formal potential of this redox process is between $-0.5 \mathrm{~V}$ and $-0.3 \mathrm{~V}$ vs. $\mathrm{Ag} / \mathrm{AgCl}[19,20]$. Thus, the redox peaks in $\mathrm{CV} 1$ indicate the formation of Ab-PSA-Ab(HRP) immuno-complex, which supports the transfer of electrons between the HRP and the electrode as observed in immuno-sensors. Alternatively, formation of Ab-PSA-Ab(HRP) immuno-complex was confirmed by testing the enzymatic activity of HRP using this electrode. CVs 2-4 in Fig. 1(d) were obtained with different concentrations of $\mathrm{H}_{2} \mathrm{O}_{2}$ in the cell. The electrode responded to the increasing $\mathrm{H}_{2} \mathrm{O}_{2}$ concentration by progressively increasing the cathodic current due to the reduction of $\mathrm{H}_{2} \mathrm{O}_{2}$ catalyzed by HRP. Note that although the CVs show weak and broadened redox peaks, the fact that the electrode's cathodic current responds to $\mathrm{H}_{2} \mathrm{O}_{2}$ indicates the enzymatic activity of HRP as previously observed [21].

In this work, two approaches were used to obtain the detection signal. The first approach required the measurement of the reduction peak current of HRP and used it as the signal. This is a reagentless method [22], in which no $\mathrm{H}_{2} \mathrm{O}_{2}$ is used to initiate the activity of HRP. To carry out the detection, cyclic voltammetry or linear voltammetry (LV) was performed with electrodes, on which the Ab-PSA-Ab(HRP) structure was formed with serum solutions containing different PSA concentrations. Specifically, the detection signal was obtained by measuring the reduction currents of the $\mathrm{Ab}-\mathrm{PSA}-\mathrm{Ab}(\mathrm{HRP})$ electrodes at about $-0.32 \mathrm{~V} \mathrm{vs}$. $\mathrm{Ag} / \mathrm{AgCl}$, 

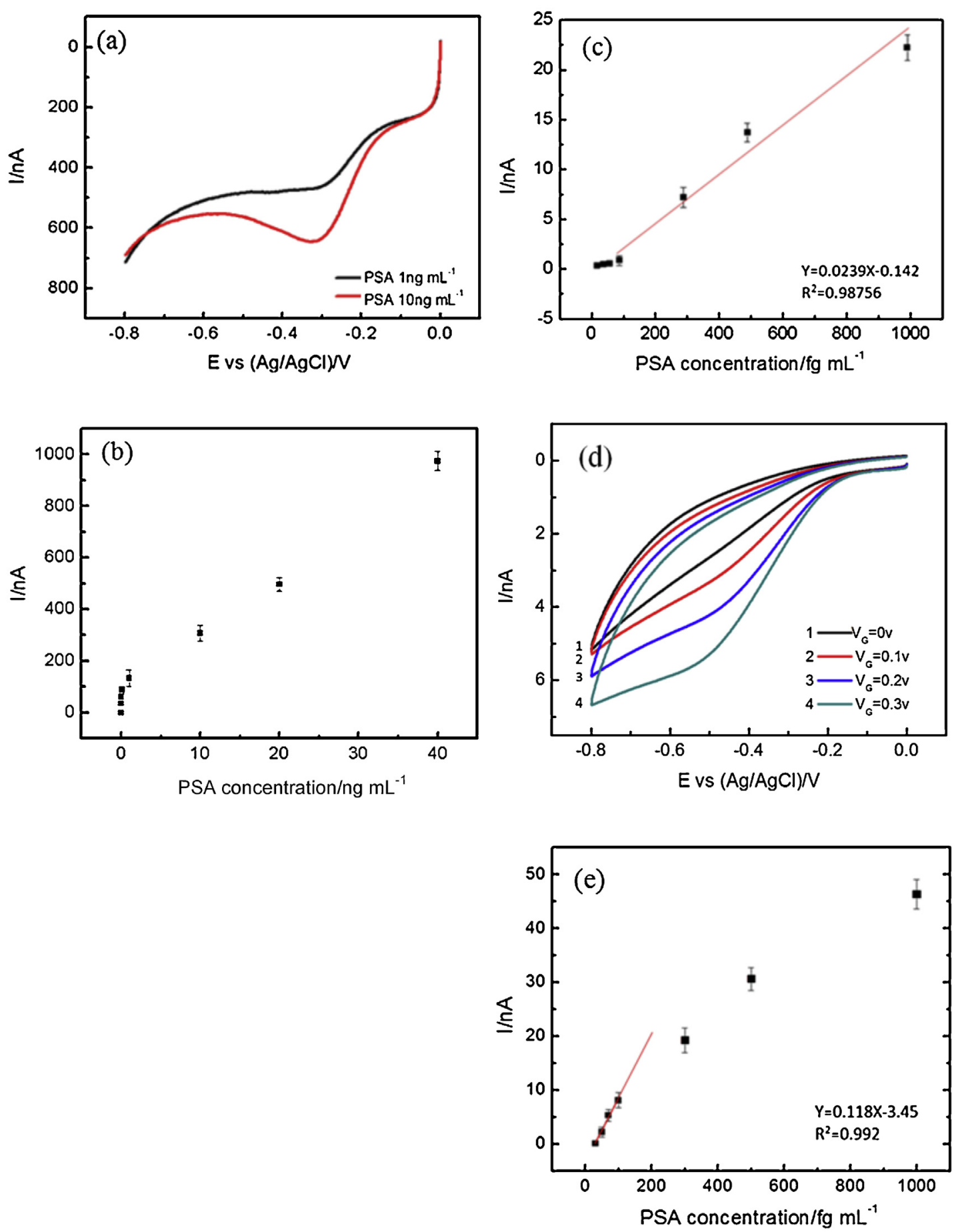

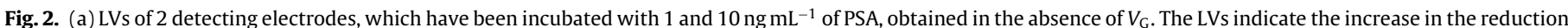

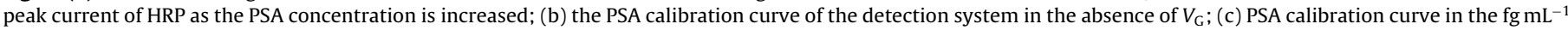

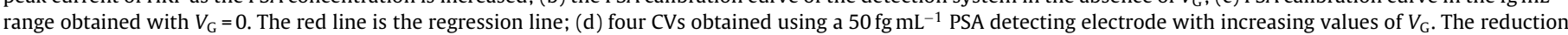

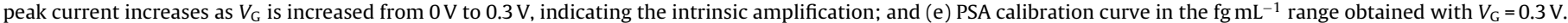
The red line is the regression line. (For interpretation of the references to color in this figure legend, the reader is referred to the web version of the article.)

where the reduction peak is located. Fig. 2(a) shows the LVs of two $\mathrm{Ab}-\mathrm{PSA}-\mathrm{Ab}(\mathrm{HRP})$ electrodes, which were incubated with different PSA concentrations ( 1 and $10 \mathrm{ng} \mathrm{mL}^{-1}$ ), obtained in the absence of $V_{\mathrm{G}}$. The LVs show that as the PSA concentration is increased, the reduction peak current increases. Fig. 2(b) shows the PSA calibration curve of the detection system obtained in the absence of $V_{\mathrm{G}}$. The data points indicate a detection range of $0.001 \mathrm{ng} \mathrm{mL}^{-1}$ ( $\left.1000 \mathrm{fg} \mathrm{mL}^{-1}\right)-40 \mathrm{ng} \mathrm{mL}^{-1}$ of PSA. The curve shows two linear regions. The high-concentration portion of the curve, i.e. the range $1-40 \mathrm{ng} \mathrm{mL} \mathrm{m}^{-1}$, exhibits a linear dependence of the signal current on PSA concentration and covers the prostate cancer screening range [23]. Below $1000 \mathrm{fg} \mathrm{mL}^{-1}$, however, the detection appears to be unsatisfactory. The calibration curve in Fig. 2(c) shows the detailed structure of the curve below $1000 \mathrm{fg} \mathrm{mL}^{-1}$. The first four data points $\left(30,50,70,100 \mathrm{fg} \mathrm{mL}^{-1}\right)$ indicate extremely low currents and their values overlap. At higher concentrations $\left(300,500,1000 \mathrm{fg} \mathrm{mL}^{-1}\right) \mathrm{a}$ 
linear region appears. The red line is the regression line for the data points, having a correlation coefficient of 0.98756 . The sensitivity is $2.3 \times 10^{-2} \mathrm{nAmL} / \mathrm{fg}$. The detection limit of this PSA concentration range was estimated to be $119 \mathrm{fg} \mathrm{mL}^{-1}$, using the signal/noise $=3$ method. The small error bars indicate reproducible device performance.

Electron transfer in amperometric immuno-sensing systems is an overlooked problem. The length of the non-electroactive $\mathrm{Ab}-\mathrm{Ag}-\mathrm{Ab}$ nanostructure is on the order of $\sim 20 \mathrm{~nm}$ for protein biomarkers. Since electron tunnel rate depends exponentially on distance as $k_{e t} \propto \exp (-\beta d)$, where $d$ is the distance through the insulating barrier between the donor and the acceptor and $\beta$ is the attenuation coefficient, which is proportional to the square root of the tunnel barrier height $\left(\beta \propto\left(\Phi_{0}\right)^{1 / 2}\right)$ [24], the length of the nanostructure is long enough to diminish the electron transfer rate and, therefore, reduce detection sensitivity. The FEED technique modifies the energy profile of the $\mathrm{Ab}-\mathrm{Ag}-\mathrm{Ab}$ nanostructure so that $\Phi_{0}$ is reduced and hence the electron transfer rate through this nanoscale interfacial region is enhanced, resulting in signal amplification and significantly lowered detection limit. This mechanism has been studied in several publications $[10,25,26]$. In particular, the effect of $V_{\mathrm{G}}$ on the reduction-peak current has been previously observed to increase as $V_{G}$ was increased [26]. Further, the same principle has been used to control electron flow in nonelectroactive protein and nanotubes [27,28]. Fig. 2(d) shows four CVs obtained using a $50 \mathrm{fg} \mathrm{mL}^{-1}$ PSA electrode with different values of $V_{\mathrm{G}}$. Fig. 2(d) shows that the reduction-peak current increases from zero to $3 \mathrm{nA}$ as $V_{\mathrm{G}}$ is increased from $0 \mathrm{~V}$ to $0.3 \mathrm{~V}$, indicating the voltage-controlled (intrinsic) amplification. The reduction peak current will keep increasing of greater $V_{G}$ values. Previously, the increase in the signal current of the FEED system, which is used in this work, caused by $V_{\mathrm{G}}$ in the positive polarity was attributed to the reduction of the height of the tunnel barrier between the active site of the enzyme and the electrode. $V_{\mathrm{G}}$ induces an electric field at the enzyme-electrode interface, and the field reduces the effective height of the tunnel barrier, resulting in an increase in the tunnel rate of electrons $[10,25,26]$. It is believed that the same mechanism is responsible for the observed increase in the signal current in the present work. A confirmation of this mechanism is provided by reversing the polarity of $V_{G}$ as shown previously $[25,26]$. The reduction peak current decreases as $V_{\mathrm{G}}$ becomes negative because the tunnel barrier becomes higher.

The calibration curve in Fig. 2(e) is the PSA calibration curve in the fg mL $\mathrm{m}^{-1}$ range obtained with $V_{\mathrm{G}}=0.3 \mathrm{~V}$, which was chosen for this concentration range. The first data point, $30 \mathrm{fg} \mathrm{mL}^{-1}$, indicates an extremely low current. Beyond that, each data point is clearly separated from others, indicating detection with $\mathrm{fg} \mathrm{mL}^{-1}$ resolution. The first four data points indicate a linear dependence of signal on PSA concentration. Higher concentration data points show a deviation from the linearity possibly due to less available binding sites or crowding. The red line is the regression line for the first four data points, having a correlation coefficient of 0.99200 . The sensitivity derived using the red line is $0.118 \mathrm{nA} \mathrm{mL} / \mathrm{fg}$. The detection limit estimated using this PSA concentration range is $27 \mathrm{fg} \mathrm{mL}^{-1}$, using the signal/noise $=3$ method. The small error bars indicate reproducible device performance. Fig. 2(c) and (e) shows that the application of $V_{\mathrm{G}}$ has increased the detection sensitivity while lowered the detection limit.

Detection of PSA was also achieved by monitoring the temporal responses of electrodes, on which the Ab-PSA-Ab(HRP) structure was formed, when $\mathrm{H}_{2} \mathrm{O}_{2}$ was injected into the cell with $V_{G}$ set to a constant value. Fig. 3(a) shows temporal traces obtained with different electrodes with the cell potential set at $-0.32 \mathrm{~V}$ and $V_{\mathrm{G}}=0.3 \mathrm{~V}$. The initial jump in the current was caused by injecting $0.1 \mathrm{mM} \mathrm{H}_{2} \mathrm{O}_{2}$ at the electrodes. The control trace, obtained with only the primary $\mathrm{Ab}$ and the labeled secondary $\mathrm{Ab}(\mathrm{HRP})$ on the
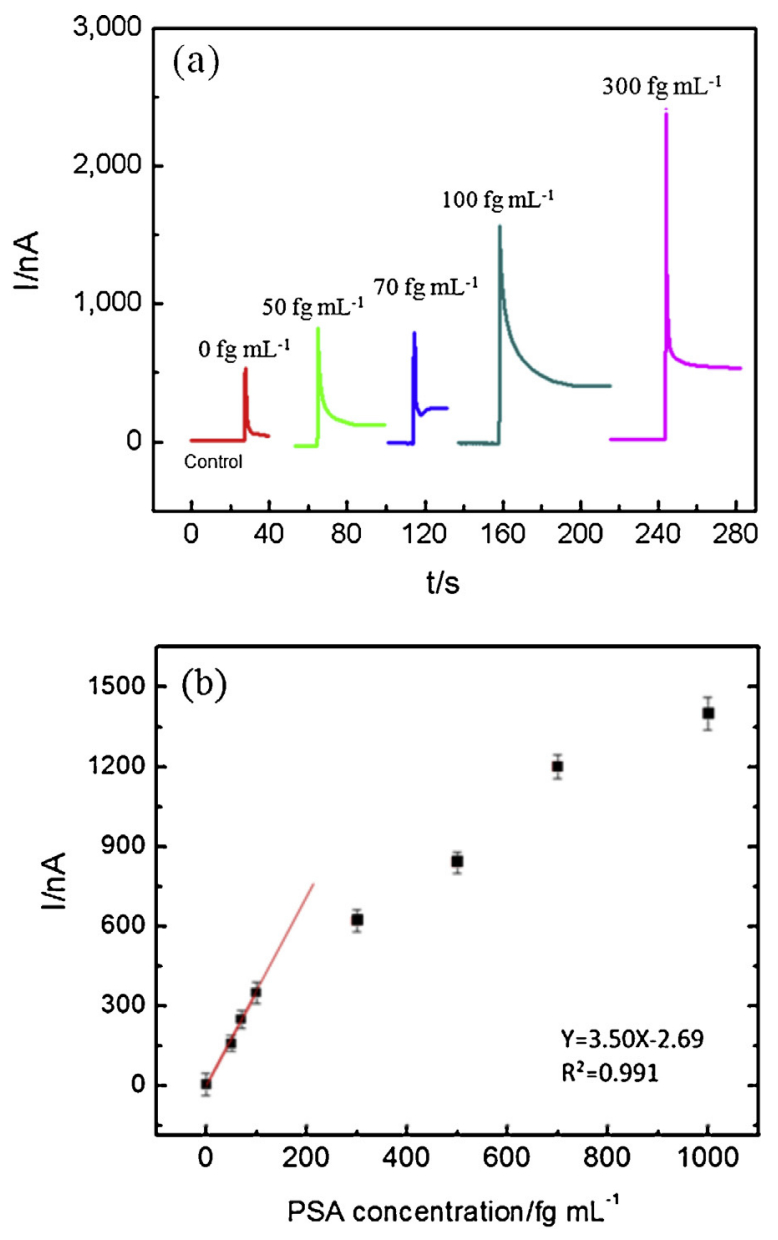

Fig. 3. (a) Temporal traces obtained with different PSA-detecting electrodes with the cell potential set at $-0.32 \mathrm{~V}$ and $V_{\mathrm{G}}=0.3 \mathrm{~V}$. The initial jumps in the current were caused by injecting $0.1 \mathrm{mM} \mathrm{H}_{2} \mathrm{O}_{2}$ near the electrodes; (b) the PSA calibration curve on the $\mathrm{fg} \mathrm{mL}^{-1}$ level based on the temporal measurements. The red line is the regression line. (For interpretation of the references to color in this figure legend, the reader is referred to the web version of the article.)

electrode, is also displayed in Fig. 3(a). The plateau of each trace was used as the detection signal since the peak current was caused by injecting $\mathrm{H}_{2} \mathrm{O}_{2}$ near the electrodes surface. Fig. 3(b) shows the PSA calibration curve on the $\mathrm{fg} \mathrm{mL}^{-1}$ level. The first four data points indicate a linear dependence of signal on PSA concentration. Higher concentration data points show a deviation from the linearity possibly due to less available binding sites or crowding. The red line is the regression line for the first four data points, having a correlation coefficient of 0.99100 . The sensitivity derived using the red line is $3.50 \mathrm{nA} \mathrm{mL} / \mathrm{fg}$. The detection limit of this PSA concentration range was estimated to be $39 \mathrm{fg} \mathrm{mL}^{-1}$, using the signal/noise $=3$ method. The small error bars indicate reproducible device performance.

Comparing Fig. 3(b) and Fig. 2 (e) shows two characteristics of the two methods for getting the detection signal. The use of $\mathrm{H}_{2} \mathrm{O}_{2}$ has significantly increased the signal current level, which might be the cause for the improved linear dependence of the signal on PSA concentration as shown in Fig. 3(b). Further, Fig. 3(b) also shows that the use of $\mathrm{H}_{2} \mathrm{O}_{2}$ has significantly enhanced detection sensitivity. However, since the noise fluctuation (zero PSA current) has also become larger due to the use of $\mathrm{H}_{2} \mathrm{O}_{2}$ and the amplification provided by the detection system, the detection limit of the $\mathrm{H}_{2} \mathrm{O}_{2}$ based amperometric method has become higher than that of the reagentless method for the same value of $V_{\mathrm{G}}$. Nevertheless, the enhanced sensitivity was able to keep the detection limit below $100 \mathrm{fg} \mathrm{mL}^{-1}$. 
The ultrasensitive detection of PSA provided by the FEED-based detection method has been demonstrated using two approaches to obtain the detection signal, showing similar detection limits on the fg $\mathrm{mL}^{-1}$ level. This capability is due to the intrinsic amplification of FEED and is illustrated using Fig. 2(c) and (e). The two calibration curves in these figures involve the same PSA concentrations. The curve in Fig. 2(c), obtained with $V_{G}=0 \mathrm{~V}$, shows a smaller current span and the four lowest concentrations yield zero current. The effect of $V_{\mathrm{G}}$ is reflected in the curve in Fig. 2(e), where each of the four lowest concentrations corresponds to a distinctive current with small error bars. The curve also shows a detection resolution of $20 \mathrm{fg} \mathrm{mL}^{-1}$ and a larger current span. The selectivity of the detection method has been demonstrated by the fact that all PSA samples used in this work, including those used in the $\mathrm{fg} \mathrm{mL}^{-1}$ level detection, were prepared with undiluted serum sample as received. Generally, serum contains a large number of biological substances such as proteins, hormones, enzymes, and antibodies. The fact that PSA detection has been successful on the $\mathrm{fg} \mathrm{mL}^{-1}$ level, where PSA samples have been diluted 1-million times using undiluted serum sample or other biological substances have a 1-million-fold higher concentration, indicates high detection selectivity. Another important property of the detection system is the limited detection of PSA below $1000 \mathrm{fg} \mathrm{mL}^{-1}$ obtained without $V_{\mathrm{G}}$. We attribute this low concentration detection capability to the immobilization of the primary antibody and hence the Ab-PSA-Ab(HRP) structure in PANI. The redox activity of PANI is known to lead to high detection sensitivity [29]. When the primary antibody is entrapped in the PANI matrix, PANI serves as an efficient non-diffusional electron mediator [15].

The ultrasensitive PSA detection technique reported here exhibits several advantages compared with the two published ultrasensitive PSA detection methods described above $[8,9]$. The PSA detection limits of $27 \mathrm{fg} \mathrm{mL}^{-1}$ and $39 \mathrm{fg} \mathrm{mL}^{-1}$ demonstrated in the present work are noticeably lower than those obtained in the two published works. The FEED-based technique with its intrinsic amplification basically requires no additional processing of reagents other than that is required to form the sandwich structure while the other two methods require extensive preparation of reagents to achieve signal amplification. Also, the FEEDbased technique employs an interfacial electric field to enhance the transfer of electrons so that no electron transfer mediators are needed in the operation of the detection system. Thus, the featured detection technique appears to be a more attractive approach for applications that demand fast detection and low-cost.

\section{Conclusions}

This work shows that FEED, when combined with the conventional amperometric immuno-sensing, can be used to detect PSA in serum with detection limit on the $\mathrm{fg} \mathrm{mL}^{-1}$ level. This capability has been demonstrated using two approaches in obtaining the detection signal, resulting in detection limit as low as $27 \mathrm{fg} \mathrm{mL} L^{-1}$. The high selectivity of the detection system is provided by the immunological reaction and is reflected in the fact that PSA detection has been successful with PSA samples that contained other biological substances having a 1-million-fold higher concentration. Note that the FEED-based immuno-sensing technique is a mediatorless approach, while most electrochemical immuno-sensors use mediators, which may lead to the leaching of the mediators out of the electrode, resulting in significant signal loss. The ultrasensitive detection and the electrochemical nature of the detection technique suggest the detection system can be integrated with microfluidics on chips for ultrasensitive analysis of small volume or low-concentration PSA samples for early detection of recurrence of prostate cancer.

\section{Acknowledgment}

This work is supported by Cleveland State University.

\section{Appendix A. Supplementary data}

Supplementary data associated with this article can be found, in the online version, at http://dx.doi.org/10. 1016/j.electacta.2013.08.046.

\section{References}

[1] T.J. Polascik, J.E. Oesterling, A.W. Partin, Prostate specific antigen: a decade of discovery what we have learned and where we are going, J. Urol. 162 (1999) 293.

[2] C. Stephan, T. Kopke, A. Semjonow, M. Lein, S. Deger, M. Schrader, K. Miller K. Jung, Discordant total and free prostate-specific antigen (PSA) assay: does calibration with WHO reference materials diminish the problem? Clin. Chem. Lab. Med. 47 (2009) 1325.

[3] G.L. Andriole, E.D. Crawford, R.L. Grubb, S.S. Buys, D. Chia, T.R. Church, M.N Fouad, E.P. Gelmann, P.A. Kvale, D.J. Reding, J.L. Weissfeld, L.A. Yokochi, B. O‘Brien, J.D. Clapp, J.M. Rathmell, T.L. Riley, R.B. Hayes, B.S. Kramer, G. Izmirlian, A.B. Miller, P.F. Pinsky, P.C. Prorok, J.K. Gohagan, C.D. Berg, Mortality results from a randomized prostate-cancer screening trial, N. Engl. J. Med. 360 (2009) 310

[4] S.J. Freedland, J.W. Moul, Prostate specific antigen recurrence after definitive therapy, J. Urol. 177 (2007) 1985.

[5] J.K. Parsons, J.K. Parsons, A.W. Partin, B. Trock, D.J. Bruzek, C. Cheli, L.J. Sokoll, Complex prostate-specific antigen for the diagnosis of biochemical recurrence after radical prostatectomy, BJU Int. 99 (2007) 758.

[6] P. Link, F.S. Freiha, T.A. Stamey, Adjuvant radiation therapy in patients with detectable prostate specific antigen following radical prostatectomy, J. Urol. 145 (1991) 532.

[7] P.H. Lange, D.J. Lightner, E. Medini, P.K. Reddy, R.L. Vessella, The effect of radiation therapy after radical prostatectomy in patients with elevated prostate specific antigen levels, J. Urol. 144 (1990) 927

[8] C.S. Thaxton, R. Elghanian, A.D. Thomas, S.I. Stoeva, J.-S. Lee, N.D. Smith, A.J. Schaeffer, H. Klocker, W. Horninger, G. Bartsch, C.A. Mirkin, Nanoparticle-based bio-barcode assay redefines "undetectable" PSA and biochemical recurrence after radical prostatectomy, Proc. Natl. Acad. Sci. U.S.A. 106 (2009) 18437.

[9] X. Yu, B. Munge, V. Patel, G. Jensen, A. Bhirde, J.D. Gong, S. Kim, J. Gillespie, J.S Gutkind, F. Papadimitraopoulos, J.F. Rusling, Carbon nanotube amplification strategies for highly sensitive immunodetection of cancer biomarkers, J. Am. Chem. Soc. 128 (2006) 11199.

[10] Y. Choi, S.-T. Yau, A field-effect enzymatic amplifying detector with pico-molar detection limit, Anal. Chem. 81 (2009) 7123

[11] A. Warsinke, Point-of-care testing of proteins, Anal. Bioanal. Chem. 393 (2009) 1393.

[12] V.V.R. Sai, S. Mahajan, A.Q. Contractor, S. Mukherji, Immobilization of antibodies on polyaniline films and its application in a piezoelectric immunosensor, Anal. Chem. 78 (2006) 8368.

[13] A.J. Killard, S. Zhang, H. Zhao, R. John, E.I. Iwuoha, M.R. Smyth, Development of an electrochemical flow-injection immunoassay for the real-time monitoring of biospecific interactions, Anal. Chim. Acta 400 (1999) 109

[14] Q. Liu, M.H. Nayfeh, S.-T. Yau, Supercapacitor electrodes based on polyaniline-silicon nanoparticle composite, J. Power Sources 195 (2010) 3956.

[15] G.R.N. Mathebe, A. Morrin, E.I. Iwuoha, Electrochemistry and scanning electron microscopy of polyaniline/peroxidase-based biosensor, Talanta 64 (2004) 115

[16] E.I. Gill, A. Arshak, K. Arshak, O. Korostynska, Investigation of thick-film polyaniline-based conductimetric $\mathrm{pH}$ sensors for medical applications, IEEE Sens. J. 9 (2009) 555

[17] A.M. Bonastre, P.N. Bartlett, Electrodeposition of PANi films on platinum needle type microelectrodes. Application to the oxidation of ascorbate in human plasma, Anal. Chim. Acta 676 (2010) 1.

[18] P. Turano, Y. Lu, Iron heme and related proteins, in: I. Bertini, A. Sigel, H. Sige (Eds.), Handbook on Metalloproteins, Marcel Dekker, New York, 2001, p. 269.

[19] T. Ferri, A. Poscia, R. Santucci, Direct electrochemistry of membrane-entrapped horseradish peroxidase: Part I. A voltammetric and spectroscopic study, Bioelectrochem. Bioeng. 44 (1998) 177

[20] Y.Zhang, P. He, N. Hu, Horseradish peroxidase immobilized in $\mathrm{TiO}_{2}$ nanoparticle films on pyrolytic graphite electrodes: direct electrochemistry and bioelectrocatalysis, Electrochim. Acta 49 (2004) 1981.

[21] R. Andreu, E.E. Ferapontova, L. Gorton, J.J. Calvente, Direct electron transfer kinetics in horseradish peroxidase electrocatalysis, J. Phys. Chem. B 111 (2007) 469. 
[22] Z. Dai, F. Yan, J. Chen, H. Ju, Reagentless amperometric immunosensors based on direct electrochemistry of horseradish peroxidase for determination of carcinoma antigen-125, Anal. Chem. 75 (2003) 5429.

[23] A.S. Brett, R.J. Ablin, Prostate-cancer screening - what the US Preventive Services Task Force left out, N. Engl. J. Med. 365 (2011) 1949.

[24] C.C. Page, C.C. Moser, X. Chen, P.L. Dutton, Natural engineering principles of electron tunnelling in biological oxidation-reduction, Nature 402 (1999) 47.

[25] Y. Choi, S.-T. Yau, Field-controlled electron transfer and reaction kinetics of the biological catalytic system of microperoxidase- 11 and hydrogen peroxide, AIP Adv. 1 (2011) 042175.
[26] J. Wang, S.-T. Yau, Field-effect amperometric immuno-detection of biomarker, Biosen. Bioelectron. 29 (2011) 210.

[27] S.-T. Yau, G. Qian, A prototype protein field-effect transistor, Appl. Phys. Lett. 86 (2005) 103508

[28] S.J. Tans, A.R.M. Verschueren, C. Dekker, Room-temperature transistor based on a single carbon nanotube, Nature 393 (1998) 49.

[29] C. Dhand, M. Das, M. Datta, B.D. Malhotra, Recent advances in polyaniline based biosensors, Biosen. Bioelectron. 26 (2011) 2811. 